\title{
Utility of intraperitoneal hyperthermic chemotherapy in peritoneal cancer from endometrial cancer - literature review
}

\author{
Nicolae BACALBASA ${ }^{1}$, Irina BALESCU ${ }^{2}$ \\ ${ }^{1}$ Department of Obstetrics and Gynecology, "Carol Davila" University of Medicine and Pharmacy, \\ Bucharest, Romania \\ ${ }^{2}$ Department of General Surgery, Ponderas Academic Hospital, Bucharest, Romania
}

\begin{abstract}
Once the benefits of intraperitoneal heated chemotherapy has been widely reported in patients presenting colorectal, appendicular or even ovarian malignancies, this method has been proposed as a therapeutic strategy in treating peritoneal carcinomatosis with various origins such as gastric cancer, breast cancer or even endometrial cancer. Although most often patients presenting endometrial cancer are diagnosed in an early stage of the disease and are submitted to surgery with curative intent, in certain cases the diagnostic is established only later in the evolution of the disease, when disseminated peritoneal lesions are present. In all these cases an aggressive surgical approach consisting to debulking surgery to no residual disease seems the best therapeutic option in order to achieve a good control of the disease. Moreover, it has been stipulated that association of intraperitoneal heated chemotherapy might improve the long term outcomes of such patients. This is a literature review regarding the utility of intraperitoneal heated chemotherapy in treating peritoneal carcinomatosis with endometrial origin.
\end{abstract}

Keywords: endometrial cancer, peritoneal carcinomatosis, intraperitoneal heated chemotherapy

\section{INTRODUCTION}

Endometrial cancer seems to be the most common gynecological cancer affecting women worldwide (1). Due to the fact that endometrial cancer rapidly conducts to the apparition of signs such as postmenopausal vaginal bleeding and pelvic pain, it is most often diagnosed in an early stage of the disease, in which the pathological process in limited to the uterus. In all these cases the patients can be safely submitted to surgery, excellent long term survival rates being reported (2). However, not all the patients are diagnosed in early stages of the disease, being estimated that up to $10 \%$ of cases will present advanced stage lesions even from the beginning (3-5). In these cases the prognostic becomes very poor, the 5 year overall survival rate being of less than 20\% (when compared with up to $80 \%$ in all patients diagnosed with endometrial cancer) (5). Due to these significant differences, attention was focused on determining which are the therapeutic strategies which might improve the long term outcomes of cases diagnosed in advanced stages of the disease.

\section{The role of cytoreductive surgery in advanced stage endometrial cancer}

Once the benefits of the cytoreductive surgery have been demonstrated in advanced stage ovarian cancer, surgeons worldwide tried to evaluate whether a similar aggressive surgical 
approach might improve the long term outcomes in patients with advanced stage endometrial cancer too $(4,6)$. However, the argumentation of debulking surgery in advanced stage endometrial cancer was more difficult to be sustained due to the reduced numbers of patients who are amenable to this type of treatment.

One of the largest studies which investigated the effectiveness of debulking surgery in endometrial cancer originates from Japan and was published in 2010 (4). The study included 31 patients submitted to surgery for advanced stage endometrial cancer and their results were compared to a similar group which was submitted to medical treatment consisting of systemic chemotherapy in six cases, hormonal therapy in two cases and radiotherapy in one case. Among patients submitted to systemic chemotherapy, surgery was associated in two cases. Therefore debulking surgery was finally administrated in 33 cases. The main associated surgical procedures included low anterior rectal resection in three cases. Optimal cytoreductive surgery was defined as residual disease less than or equal to one $\mathrm{cm}$ and was achieved in 23 cases. The authors reported a median disease free survival for the whole group of 10.4 months and a median overall survival of 21.3 months. The overall survival was significantly influenced by the degree of differentiation and by the presence of extraperitoneal metastases as well as by the administration of the hormonal therapy; surprisingly, association of chemotherapy, radiotherapy or achieving an optimal debulking surgery did not significantly impact on the overall survival. The multivariate analysis also demonstrated that the most important prognostic factors were the degree of differentiation and the number of the extraperitoneal lesions while cytoreductive surgery did not impact on the overall survival. Moreover the authors demonstrated that among long term survivors all cases presented no more than one extraperitoneal lesions; in the meantime five of the seven short term survivors reported at least two extraperitoneal lesions. These data enabled the authors to conclude that the biological aggressiveness has a higher impact on the long term outcomes of these patients when compared to the completeness of the cytoreduction (4). However the study had few limitations regarding its retrospective nature as well as the small number of included patients.

Not at the same conclusion got another Japanese study published in the same year in An- nals of Surgical Oncology. This time the authors included in their study 33 patients with stage IVB endometrial cancer, 30 of them being submitted to cytoreductive surgery. The most commonly reported sites presenting distant metastases included the retroperitoneal lymph nodes, the abdominal peritoneum, the omentum, the bowel serosa or mesentery as well as the liver.

This time the optimal cytoreductive surgery was defined by the presence of a residual tumor lesser than $2 \mathrm{~cm}$; the authors demonstrated that achieving an optimal cytoreduction significantly improved the outcomes of these patients. The most commonly extrapelvic resections included omentectomies, peritonectomies, small bowell resections, liver resections as well as lung resections. The authors reported that the median overall survival was significantly influenced by the age of the patient, by the histopathological subtype and by the effectiveness of the debulking procedure. The median overall survival in patients submitted to optimal cytoreductive surgery in the absence of extraabdominal metastases was 43 months, significantly higher when compared to the one reported after suboptimal cytoreduction (with a reported overall survival of only 6 months, $p=0.0007$ ). Moreover, the authors studied the influence of the optimal cytoreductive surgery in patients presenting also extraabdominal lesions and demonstrated that the completeness of cytoreduction had a significant impact on the overall survival in these cases too. These results were also confirmed by the multivariate analysis (6).

Other important studies demonstrating the effectiveness of cytoreductive surgery in endometrial cancer were conducted by Bristow (7), Ayhan (8) and Chi (9).

However, an interesting aspect revealed by the study conducted by Ueda et al. demonstrated the best outcomes were obtained in cases in which the optimal residual disease was lesser than $2 \mathrm{~cm}$ and not lesser than $1 \mathrm{~cm}$. Moreover, the authors demonstrated that the dimension of the residual tumor was more significant when predicting the long term outcomes when compared to the extent of the metastatic lesions (6).

\section{Association between HIPEC and debulking surgery in endometrial cancer}

In order to improve the outcomes reported after debulking surgery for advanced stage endometrial cancer, some studies investigated whether association of the intraperitoneal route of the chemotherapic administration might im- 
prove the long terms outcomes of these patients. The procedure has been widely implemented under the abbreviation of HIPEC and is represented by the intraperitoneal administration of the heated chemotherapeutic agent. The procedure seems to be more efficient in comparison with the classical, intravenous route of administration of the cytotoxic agents due to the close contact between the drug and the peritoneal surfaces. In this way the cytotoxic agent will penetrate directly the peritoneal structures at a medium depth of 3-5 mm, inducing in this way the lysosomal activation as well as the development of intracellular acidosis and subsequent cellular apoptosis. Moreover, it seems that heating the chemotherapeutic agent to 41 to $43^{\circ} \mathrm{C}$ offers a supplemental benefit, heat and cytotoxic agents having a synergic effect. Another important advantage of the technique consists in the limitation of the risk of developing severe systemic adverse reactions; using the peritoneal route of administration, a lower absorbtion in the systemic circulation is obtained, limiting in this way the rate of apparition of systemic adverse events (10-18).

Although all these data seem very promising especially for patients with peritoneal macroscopic or microscopic lesions with various origins, it should not be omitted the fact that HIPEC is an aggressive procedure which might increase the perioperative morbidity. The most commonly reported surgical related complications after administration of HIPEC consist in the apparition of various leaks, intraabdominal abscesses or postoperative hemoperitoneum (1013).

Moreover, an attentive selection of the patients is mandatory; therefore, not all the patients diagnosed with peritoneal carcinomatosis from various origins are considered eligible for this procedure. Only patients younger than 70 years, with a good general and biological status should be submitted to such procedures. Moreover, the extent of the disease plays a crucial role in identifying the patients who could be submitted to HIPEC. According to Sugarbaker, only patients with a low peritoneal carcinomatosis index should be included for HIPEC. Another essential condition in order to be eligible for HIPEC is represented by the completeness of the cytoreduction; it has been proven that only cases in which an optimal cytoreduction had been achieved during the cytoreductive phase are candidates for HIPEC. Moreover, it seems that association of HIPEC to incomplete cytore- duction will lead to an increased risk of postoperative complications, in the absence of any benefit in regard with the long term outcomes (12-18).

When it comes to the effectiveness of HIPEC in patients with peritoneal carcinomatosis with endometrial cancer, promising results have been reported so far especially if the cytotoxic drug is a platinum based salt (18).

In a study conducted at "Gustave Roussy" Cancer Center in Paris and published in 2016 the authors investigated the role of debulking surgery in association with HIPEC in peritoneal carcinomatosis with unusual origin. Among the 31 reviewed cases the authors reported one patient with peritoneal cancer from endometrial origin in a patient who had been initially diagnosed with serous endometrial cancer. Twenty-four months later the patient was diagnosed with peritoneal carcinomatosis, having a peritoneal carcinomatosis index of 19. At that moment the case was submitted to debulking surgery and HIPEC with good results; at a 28 month follow up the patient being free of disease (19).

One of the largest studies which focused on the role of HIPEC in association with debulking surgery for peritoneal carcinomatosis with endometrial origin was published in the European Journal of Obstetrics, Gynecology and Reproductive Biology in 2014 (20). The study included 13 patients submitted to debulking surgery and HIPEC between 2001 and 2013 in Nice, France. In all cases the initial extent of the disease was established after calculating the peritoneal carcinomatosis index while the completeness of cytoreduction was estimated using a completeness cytoreduction score (CC-S). According to these authors, CC-S was 0 if no macroscopic residual disease was seen at the end of surgery, 1 if the diameter of the largest tumor was lesser than $2.5 \mathrm{~mm}, 2$ if the diameter of the largest tumor ranged between $2.5 \mathrm{~mm}$ and $25 \mathrm{~mm}$ and 3 if the diameter of the residual tumor surpassed $25 \mathrm{~mm}$. In all cases the HIPEC procedure consisted in the administration of heated cisplatin. Among the 13 cases diagnosed with peritoneal carcinomatosis from endometrial origin four of them were submitted to HIPEC at the time of diagnostic while the other nine cases were submitted to HIPEC at the moment of the diagnostic of recurrence. At the end of the cytoreductive surgery the CC-S score was 0 for eight cases, 1 for three cases and 2 in the last two patients. Postoperatively a single patient developed a grade II complication. When it comes to the 
long term outcomes, the median overall survival was 19,4 months while the disease free survival was 11.4 months (range 1.5-124 months) (20). Therefore the authors underlined that association of HIPEC to the debulking surgery for endometrial cancer peritoneal carcinomatosis might significantly improve the long term outcomes especially if cytoreductive surgery to no residual disease is achieved (20).

\section{CONCLUSIONS}

Although reported data is scarce, it seems that association between HIPEC and debulking surgery can significantly improve the long term outcomes in patients with peritoneal carcinomatosis from endometrial cancer. It seems that the best results are obtained after the intraperitoneal administration of heated cisplatin especially if no residual disease is found at the end of the resectional phase. However, it should not be omitted that this procedure might induce the apparition of significant postoperative complications; therefore a careful selection of the patients is mandatory.

Conflict of interest: none declared Financial support: none declared

\section{REFERENCES}

1. Jemal A., Murray T., Samuels A., Ghafoor A., Ward E., Thun M.J. Cancer statistics, 2003. CA Cancer J Clin 53: 5-26, 2003.

2. Sanjuan A., Cobo T., Escaramis G., Rovirosa A., Ordi J., Garcia S., Hernandez S., Caparros X., Torne A., Martinez R.S., Lejarcegui J.A., Pahisa J. Recurrence and survival in surgically treated endometrioid endometrial cancer Clin Transl Oncol 10: 505-511, 2008.

3. Annual report on patients with endometrial cancer in 2005. Gynecologic Cancer Committee in Japan Society of Obstetrics and Gynecology ed. 2005.

4. Tanioka M., Katsumata N., Sasajima Y., Ikeda S., Kato T., Onda T., Kasamatsu T., Fujiwara Y. Clinical characteristics and outcomes of women with stage IV endometrial cancer. Med Oncol 27: 1371-1377, 2010.

5. Creasman W.T., Odicino F., Maisonneuve P., Quinn M.A., Beller U., Benedet J.L., Heintz A.P., Ngan H.Y., Pecorelli S. Carcinoma of the corpus uteri. FIGO 26th Annual Report on the Results of Treatment in Gynecological Cancer. Int J Gynaecol Obstet 95 Suppl 1: S105-S143, 2006.

6. Ueda Y., Enomoto T., Miyatake T., Egawa-Takata T., Ugaki H., Yoshino K., Fujita M., Kimura T. Endometrial carcinoma with extra-abdominal metastasis: improved prognosis following cytoreductive surgery. Ann Surg Oncol 17: 1111-1117, 2010.

7. Bristow R.E., Tomacruz R.S., Armstrong D.K., Trimble E.L., Montz F.J. Survival effect of maximal cytoreductive surgery for advanced ovarian carcinoma during the platinum era: a meta-analysis. J Clin Oncol 20: 1248-1259, 2002.

8. Ayhan A., Taskiran C., Celik C., Yuce K., Kucukali T. The influence of cytoreductive surgery on survival and morbidity in stage
IVB endometrial cancer. Int J Gynecol Cancer 12: 448-453, 2002.

9. Chi D.S., Welshinger M., Venkatraman E.S., Barakat R.R. The role of surgical cytoreduction in Stage IV endometrial carcinoma. Gynecol Oncol 67: 56-60, 1997.

10. Franko J., Shi Q., Goldman C.D., Pockaj B.A., Nelson G.D., Goldberg R.M., Pitot H.C., Grothey A., Alberts S.R., Sargent D.J. Treatment of colorectal peritoneal carcinomatosis with systemic chemotherapy: a pooled analysis of north central cancer treatment group phase III trials N9741 and N9841. J Clin Oncol 30: 263-267, 2012.

11. Klaver Y.L., Lemmens V.E., Creemers G.J., Rutten H.J., Nienhuijs S.W., de Hingh I.H. Population-based survival of patients with peritoneal carcinomatosis from colorectal origin in the era of increasing use of palliative chemotherapy. Ann Oncol 22: 2250-2256, 2011.

12. Bernards N., Creemers G.J., Nieuwenhuijzen G.A., Bosscha K., Pruijt J.F., Lemmens V.E. No improvement in median survival for patients with metastatic gastric cancer despite increased use of chemotherapy. Ann Oncol 24: 3056-3060, 2013.

13. Sugarbaker P.H., Stuart O.A. Pharmacokinetic and phase II study of heated intraoperative intraperitoneal melphalan. Cancer Chemother Pharmacol 59: 151-155, 2007.

14. Chua T.C., Yan T.D., Saxena A., Morris D.L. Should the treatment of peritoneal carcinomatosis by cytoreductive surgery and hyperthermic intraperitoneal chemotherapy still be regarded as a highly morbid procedure?: a systematic review of morbidity and mortality. Ann Surg 249: 900-907, 2009.
15. Aarts F., Bleichrodt R.P., de Man B., Lomme R., Boerman O.C., Hendriks T. The effects of adjuvant experimental radioimmunotherapy and hyperthermic intraperitoneal chemotherapy on intestinal and abdominal healing after cytoreductive surgery for peritoneal carcinomatosis in the rat. Ann Surg Oncol 15: 3299-3307, 2008.

16. Pelz J.O., Doerfer J., Decker M., Dimmler A., Hohenberger W., Meyer T. Hyperthermic intraperitoneal chemoperfusion (HIPEC) decrease wound strength of colonic anastomosis in a rat model. Int J Colorectal Dis 22: 941-947, 2007.

17. De Somer F., Ceelen W., Delanghe J., De Smet D., Vanackere M., Pattyn P., Mortier E. Severe hyponatremia, hyperglycemia, and hyperlactatemia are associated with intraoperative hyperthermic intraperitoneal chemoperfusion with oxaliplatin. Perit Dial Int 28: 61-66, 2008.

18. Braam H.J., Schellens J.H., Boot H., van Sandick J.W., Knibbe C.A., Boerma D., van Ramshorst B. Selection of chemotherapy for hyperthermic intraperitoneal use in gastric cancer. Crit Rev Oncol Hematol 95: 282-296, 2015.

19. Honore C., Goere D., Macovei R., Colace L., Benhaim L., Elias D. Peritoneal carcinomatosis from unusual cancer origins: Is there a role for hyperthermic intraperitoneal chemotherapy? J Visc Surg 153: 101-107, 2016.

20. Delotte J., Desantis M., Frigenza M., Quaranta D., Bongain A., Benchimol D., Bereder J.M. Cytoreductive surgery with hyperthermic intraperitoneal chemotherapy for the treatment of endometrial cancer with peritoneal carcinomatosis. Eur J Obstet Gynecol Reprod Biol 172: 111-114, 2014. 